\title{
Stage one endometrioid endometrial adenocarcinoma: is there a role of traditional hospital follow-up in the detection of cancer recurrence in women after treatment?
}

\author{
Manolis Nikolopoulos, MBBS, MRCOG ${ }^{1}$, Michelle A.L. Godfrey, MBBS, MRCOG ${ }^{2}$, Fareeda Sohrabi, MBBS ${ }^{3}$, \\ Min Wong, MBBS, MRCOG ${ }^{3}$, Deepali Bhatte, MBBS, MRCOG ${ }^{3}$, Rekha Wuntakal, MBBS, MRCOG ${ }^{3}$ \\ Department of Gynaecological Oncology, ${ }^{1}$ Epsom and St Helier University Hospitals \& NHS Trust, St Helier Hospital, London; ${ }^{2}$ Queen Alexandra \\ Hospital, Portsmouth; ${ }^{3}$ Barking, Havering and Redbridge University Hospitals \& NHS Trust, Queen's Hospital, London, UK
}

\section{Objective}

To investigate the rate of asymptomatic recurrence of stage 1 endometrioid endometrial cancer and assess the role of routine hospital follow-up after treatment.

\section{Methods}

We performed a retrospective case-note review study of women who were diagnosed with stage 1 endometrioid endometrial adenocarcinoma at Queen's Hospital, Romford, between January 2008 and December 2016.

\section{Results}

We included 299 patients with a median follow-up period of 44.4 months. All the patients underwent total hysterectomy and bilateral salpingo-oophorectomy. Adjuvant radiotherapy was offered to the patients subsequent to discussions in the multidisciplinary team meeting in accordance with the risk stratification criteria. There was no significant correlation between the risk factors and disease recurrence. In total, 11 patients presented with recurrent disease with original staging: $1 a, n=6 / 199$; and $1 b, n=5 / 100$. Four patients presented with vaginal bleeding due to vault recurrence and one patient with abdominal pain due to pelvic mass. Locoregional recurrence was an incidental finding in two other patients. Four patients presented with symptomatic distant metastases to the lung $(n=2)$, liver $(n=1)$, and bone $(n=1)$. No asymptomatic recurrences were identified on routine follow-ups, despite several hospital appointments and clinical examinations. The recurrence rate for patients with stage $1 \mathrm{a}$ and $1 \mathrm{~b}$, grade 1, and grade 2 disease was $3.53 \%$, and that for patients with stage $1 \mathrm{a}$, grade 1 , and grade 2 disease was $2.7 \%$.

\section{Conclusion}

Routine clinical examinations have a low yield in finding recurrence in asymptomatic women and should be questioned for their value, considering the limited resources of the National Health Service (NHS). Larger studies are required to support a stratified follow-up, which will include telephone and patient-initiated follow-up.

Keywords: Endometrial cancer; Recurrence; Gynecology; Physical examination

Received: 2021.04.18. Revised: 2021.06.23. Accepted: 2021.08.17.

Corresponding author: Manolis Nikolopoulos, MBBS, MRCOG

Department of Gynaecological Oncology, Epsom and St Helier University Hospitals \& NHS Trust, St Helier Hospital, Wrythe Lane,

Carshalton, Sutton, London SM5 1AA, UK

E-mail: manolisnikolopoulos@gmail.com

https://orcid.org/0000-0001-5805-4253

Articles published in Obstet Gynecol Sci are open-access, distributed under the terms of the Creative Commons Attribution Non-Commercial License (http://creativecommons.org/ licenses/by-nc/3.0/) which permits unrestricted non-commercial use, distribution, and reproduction in any medium, provided the original work is properly cited. 


\section{Obstetrics \& Gynecology Science}

Manolis Nikolopoulos, et al. Endometrial cancer follow-up

\section{Introduction}

Endometrial cancer is the most common gynecological cancer in the developed world, and its increasing incidence $[1,2]$ is attributed to an increase in the prevalence of obesity and life expectancy [3]. The follow-up of patients after the treatment of endometrial cancer has historically involved outpatient appointments once in every 3-4 months for the first year after treatment, followed by appointments at 6 -month intervals for the next 2-4 years $[4,5]$. The followup involves consultation with a doctor and abdominal and pelvic examinations to identify the signs of cancer recurrence $[4,5]$. However, this follow-up strategy does not result in an improved survival benefit [6] and is therefore not considered cost-effective [7]. It is predicted that there will be 4 million cancer survivors in the UK alone by 2030 [8], leading to concerns about the sustainability of a hospital-based follow-up strategy. The National Health Service (NHS) is struggling to cater to the demands of an expanding elderly population, necessitating reforms in the traditional oncology follow-up by decreasing the number of face-to-face hospital follow-up appointments, thereby allowing the reallocation of limited resources. The current follow-up practice also does not meet all the needs of cancer survivors [9], and the National Cancer Survivorship Initiative (NCSI) for England supports a stratified model of follow-up, emphasizing supported patient selfmanagement and early patient recognition in case of disease relapse [10].

Low-grade stage 1 endometrioid endometrial adenocarcinoma has a quoted recurrence rate of $1-3 \%$, and it is uncommon for recurrences to be asymptomatic and detected solely on follow-up examination [11-13].

We present a comprehensive retrospective review of all the patients who were treated surgically for stage 1 endometrioid endometrial cancer and had their diagnosis at our gynecological oncology unit between January 2008 and December 2016. This study aimed to ascertain the clinical presentation of disease recurrence and evaluate the role of routine hospital follow-up in the detection of cancer recurrence, motivated by the favorable impact of an increasing number of hospitals in the UK adopting patient-initiated follow-up of cancer patients.

\section{Materials and methods}

\section{Patients and study design}

All the patients who had stage 1 endometrioid adenocarcinoma of the endometrium, diagnosed at Queen's Hospital, Romford, UK, from January 2008 to December 2016, were identified from the pathology database. Women with other histological types of cancer of the endometrium, such as serous, clear cell, and carcinosarcoma, were excluded. Patients who were not treated surgically were excluded. This review of clinical service provision/audit (as defined by the Health Research Authority) was registered at the Queen's Hospital Clinical Audit Department.

Data was extracted from online medical records, written case notes, histology, and radiology reports. Demographics and risk factors included age, ethnicity, body mass index (BMI), medical comorbidities of hypertension, diabetes, previous history of breast cancer, and tamoxifen use. Clinical presentation and tumor data (endometrial thickness, histology results, staging) were obtained, along with the number of routine follow-up appointments attended, clinical presentation of recurrence including the treatment received by these patients, and the outcome.

Preoperative staging was based on magnetic resonance imaging (MRI) findings and confirmed by histology. Lowrisk groups (grade 1-2, presumed stage 1a on imaging and grade 1 , and presumed stage $1 \mathrm{~B}$ on imaging) underwent surgery at Queen's hospital. High-risk groups (grade 2 and presumed stage $1 \mathrm{~B}$ ) on preoperative imaging or any grade 3 endometrial endometrioid adenocarcinoma) were treated surgically at the referral gynecological oncology center and received postoperative follow-up at Queen's hospital. Pelvic lymphadenectomy was not routinely performed according to our protocol. If the MRI pelvis showed enlarged lymph nodes or lymphadenopathy (suspicion of involvement), the lymph nodes were removed surgically. According to risk stratification (low risk, intermediate risk, and high risk), the patients were offered adjuvant radiotherapy (external beam radiotherapy, adjuvant brachytherapy, or both), depending on the final histological stage, the grade, and the presence of adverse prognostic factors, such as lymphovascular space invasion, after discussion in a multidisciplinary team (MDT) meeting. Immunohistochemistry was not a part of the risk stratification process during the time period that is being examined in the present study. 


\section{Obstetrics \& Gynecology Science}

Vol. 64, No. 6, 2021

All the patients treated for stage $1 \mathrm{a}$, grade 1 endometrioid endometrial adenocarcinoma were offered 3-5 years of hospital follow-up, entailing an appointment with a clinician every 4 months for the first year and every 6 months thereafter, as per the hospital policy. The appointment routinely involved questioning about the symptoms and clinical examination including that of the pelvis and the abdomen. Further investigations were requested if recurrence was suspected. Patients with stage IB or high-grade disease were offered the same follow-up regime for 5 years, and then transitioned to the purview of their general practitioner.

\section{Statistical methods}

Data was analyzed using IBM SPSS Statistics Version 24.0.0.0 for Macintosh "macOS" (IBM Corp., Armonk, NY, USA). Uni- variate analyses of the time to recurrence were performed with the time of first treatment as the entry date and the occurrence of recurrence, defined as the event, as the date of the follow-up appointment with clinical evidence of recurrence (symptoms/signs of recurrent disease). Fisher's exact test was used for comparisons between categorical variables, and in this case, the presumable risk factors divided into categories were considered as independent variables, and the dependent variables were defined as recurrence or not. Multivariate analyses were also performed to predict recurrence in different groups that have been considered important in the literature for predicting recurrence, specifically the stage and grade of the primary disease. The mean and median values were calculated. $P<0.05$ was considered to be statistically significant.

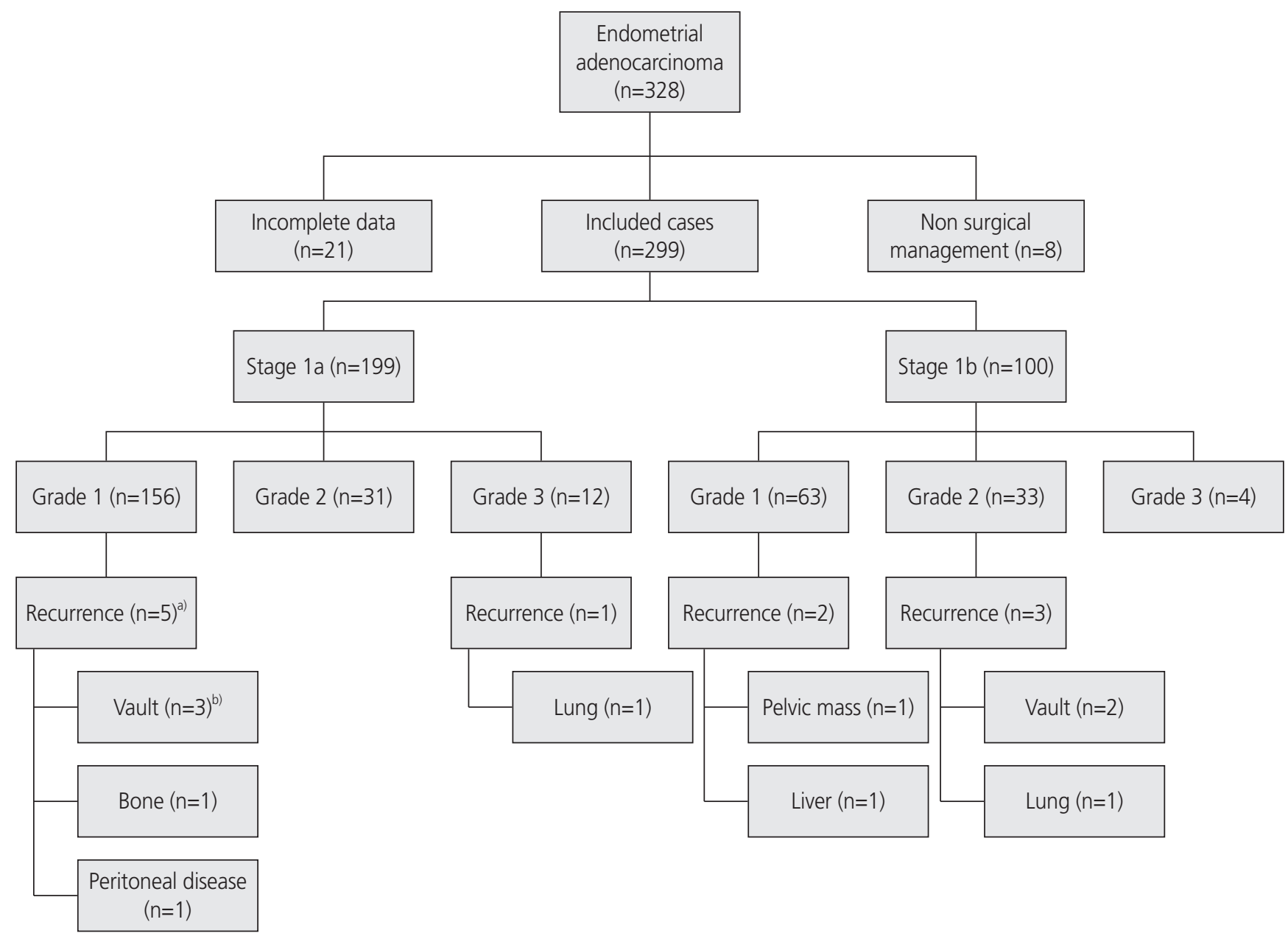

Fig. 1. Study flow chart of the 328 women with stage 1 endometrioid adenocarcinoma of the endometrium. ${ }^{\text {a) }}$ One patient's original pathology report was upgraded to grade 2 after recurrence; ${ }^{b}$ One woman with vault recurrence had lung metastasis at the time of diagnosis of recurrence. 


\section{Obstetrics \& Gynecology Science}

Manolis Nikolopoulos, et al. Endometrial cancer follow-up

\section{Results}

Over a period of 9 years, 328 patients were diagnosed with stage 1 endometrioid endometrial adenocarcinoma of various grades (Fig. 1). Of the 328 patients, 8 were treated non-surgically; therefore, they were excluded. Twenty-one patients were excluded for incomplete data identification. The remaining 299 patients underwent surgery with curative intent (total hysterectomy and bilateral salpingo-oophorectomy) and were included in the study. Preoperative crosssectional imaging was performed in 297 patients; 2 of them had endometrial cancer diagnosed after vaginal hysterectomy for prolapse and subsequently underwent laparoscopic salpingo-oophorectomy for completion of the treatment.

The mean age at diagnosis was 62 years (range 32-91 years). In total, 32 patients died: 6 due to endometrial cancer, 18 due to other known causes, and the cause of death remained unidentified in eight patients. Out of these eight patients, 5 were still under follow-up with no evidence of disease recurrence, and the remaining 3 had completed follow-up for endometrial cancer and were discharged. The median follow-up period for the cohort was 44.4 months (range 7.2-90.4 months).

Characteristics of the patients are described in Table 1. Majority of the patients were post-menopausal at diagnosis $(n=259,86.6 \%)$, and 231 of them presented with vaginal bleeding (77.3\%). Pre-menopausal women most commonly presented with irregular vaginal bleeding. Risk factors for endometrial cancer were prevalent in the cohort: $54.8 \%$ of the patients were obese, with a $\mathrm{BMI}>30,53.5 \%$ of them were hypertensive, and $19.1 \%$ of them were diabetic. There was a low prevalence $(3.7 \%)$ of "ever use" of tamoxifen.

Most patients ( $n=199,66.6 \%)$ had stage 1a disease, and 100 of them (33.4\%) had stage $1 \mathrm{~b}$ disease. Grade 1 cancer was seen in 219 patients, while 64 patients had grade 2 cancer. Of the 16 patients with grade 3 cancer, one patient had recurrence. Adjuvant radiotherapy was recommended for 81 patients following an MDT discussion. Of these, 1 patient declined radiotherapy (stage 1a, grade 3 ) and another patient was unfit for radiotherapy due to multiple comorbidities. Both these patients experienced recurrence of the disease.

Recurrence of endometrial cancer was seen in 11 patients (3.7\%) and the details are described in Table 2. Recurrence of endometrial cancer was seen in 11 patients (3.7\%). The overall recurrence rate for patients with stage 1 (1a and $1 \mathrm{~b})$ grade 1 and 2 cancers was $3.53 \%$, while among the 187 patients with stage 1 a grade 1 and 2 disease, five patients were diagnosed with recurrence $(2.7 \%)$. No recurrence was an unexpected finding on routine clinical examination during follow-up in asymptomatic patients. In two out of the $11(18 \%)$ cases of recurrence, patients waited for a few weeks for their routine clinic appointment to discuss their new symptoms.

Symptoms at presentation usually correlate with the site of recurrence. Four women presented with signs and symptoms of distant recurrence: shortness of breath due to lung metastases $(n=2)$, severe hip pain due to bone metastasis $(n=1)$, and jaundice due to liver metastases $(n=1)$. Almost all the women presenting with distant metastases died of disease progression. A woman who presented with a solitary lung metastasis, 50 months after primary surgery for stage 1a grade 1 endometrial cancer and underwent left upper lobectomy, was still in remission with letrozole hormonal treatment at the time of the last follow up appointment.

Four women experienced vaginal bleeding due to vault recurrence. Three of them presented with vaginal bleeding and had contacted the department, and one was experiencing vaginal bleeding but waited for a routine clinic appointment to discuss her symptoms. Three of the women with vaginal vault recurrence received treatment with curative intent, and all of them had further relapse several months later (range 18-32 months). One woman had concurrent gastric cancer and died later.

One woman presented with abdominal pain, bloating, and fatigue, with a computerised tomography (CT) scan revealing a pelvic peritoneal mass, enlarged lymph nodes, and a hydroureter. This case was identified on follow-up in a symptomatic patient who waited for a routine follow-up appointment to report her new symptoms.

Recurrence was an incidental finding in two women. One woman was admitted with stroke and had CT of the chest, abdomen, and pelvis, with peritoneal malignancy in the pelvis, which was presumed to be a recurrence of endometrial cancer. Finally, vaginal vault recurrence was an incidental finding on MRI performed in a woman with hip pain.

The median time from treatment to the first recurrence was 22 months (range 5-60 months post-treatment). The mean time was 28.5 months. There was no significant correlation between the risk factors and disease recurrence or 


\section{Obstetrics \& Gynecology Science}

Vol. 64, No. 6, 2021

Table 1. Characteristics of 299 women with surgically treated stage 1 endometrial cancer at Queen's hospital, from January 2008 to December 2016

\begin{tabular}{|c|c|}
\hline Characteristic & Value \\
\hline \multicolumn{2}{|l|}{ Parity } \\
\hline Nulliparous & $54(18.1)$ \\
\hline Parous & $236(78.9)$ \\
\hline Unknown & $9(3)$ \\
\hline \multicolumn{2}{|l|}{ Menopausal status } \\
\hline Pre-menopausal & $36(12.1)$ \\
\hline Post-menopausal & $259(86.6)$ \\
\hline Unknown & $4(1.3)$ \\
\hline \multicolumn{2}{|l|}{$\mathrm{BMl}>30$} \\
\hline Yes & $164(54.8)$ \\
\hline No & $71(23.7)$ \\
\hline Unknown & $55(21.5)$ \\
\hline \multicolumn{2}{|l|}{ Diabetic } \\
\hline Yes & $57(19.1)$ \\
\hline No & $197(65.9)$ \\
\hline Unknown & $40(15)$ \\
\hline \multicolumn{2}{|l|}{ Hypertensive } \\
\hline Yes & $161(53.8)$ \\
\hline No & $111(37.1)$ \\
\hline Unknown & $28(9.1)$ \\
\hline History of breast cancer & $15(5.0)$ \\
\hline Use of tamoxifen & $11(3.7)$ \\
\hline \multicolumn{2}{|l|}{ HRT } \\
\hline Current use of HRT & $12(4.0)$ \\
\hline Past use of HRT & $25(8.4)$ \\
\hline Never used & $164(44.8)$ \\
\hline Non-applicable & $36(12.0)$ \\
\hline Unknown & $92(30.8)$ \\
\hline \multicolumn{2}{|l|}{ Presentation of original disease } \\
\hline Post-menopausal bleeding & $231(77.3)$ \\
\hline Irregular bleeding/menorrhagia & $34(11.4)$ \\
\hline Vaginal discharge & $7(2.3)$ \\
\hline Incidental finding & $7(2.3)$ \\
\hline Weight loss & $2(0.6)$ \\
\hline Abdominal pain & $3(1.0)$ \\
\hline Unknown & $15(5.1)$ \\
\hline \multicolumn{2}{|l|}{ Stage } \\
\hline $1 \mathrm{a}$ & 199 (66.5) \\
\hline $1 b$ & $100(33.5)$ \\
\hline
\end{tabular}

Table 1. Continued

\begin{tabular}{lc}
\hline Characteristic & Value \\
\hline Grade & $219(73.2)$ \\
1 & $64(21.4)$ \\
2 & $16(5.4)$ \\
3 & \\
Primary surgery & $147(49.2)$ \\
$\quad$ Laparoscopic hysterectomy \& bilateral \\
salpingo-oophorectomy \\
$\begin{array}{l}\text { Abdominal hysterectomy \& bilateral } \\
\text { salpingo-oophorectomy }\end{array}$ \\
$\begin{array}{l}\text { Vaginal hysterectomy +second procedure } \\
\text { bilateral salpingo-oophorectomy }\end{array}$ \\
$\begin{array}{l}\text { Adjuvant treatment } \\
\text { Intravaginal radiotherapy }\end{array}$ \\
$\begin{array}{l}\text { External beam radiotherapy } \\
\text { Intravaginal and external radiotherapy }\end{array}$ \\
$\begin{array}{l}\text { Intravaginal radiotherapy and } \\
\text { chemotherapy) }\end{array}$ \\
$\begin{array}{l}\text { Chemotherapy) } \\
\text { None }\end{array}$ \\
$\begin{array}{l}\text { Radiotherapy declined by patient } \\
\text { Patient unfit for radiotherapy }\end{array}$ \\
\hline
\end{tabular}

Values are presented as number (\%).

BMI, body mass index; HRT, hormone replacement therapy.

a)Patient had presumed involved pelvic lymph node and received adjuvant chemo-radiotherapy; ${ }^{b}$ Patient had concurrent ovarian cancer and received adjuvant chemotherapy, despite grade 1 stage $1 a$ endometrial cancer.

between the risk factors and the time between treatment and recurrence. Age, parity, menopausal status, BMI, past and current use of hormone replacement therapy, history of diabetes, and hypertension were included as the risk factors. The history of breast cancer, tamoxifen use, and the grade and stage of primary cancer were examined, and no statistically significant difference was found. The correlation of risk factors with recurrence was not within the scope of the current study and would require more detailed analysis to arrive at definite conclusions.

\section{Discussion}

Our study found that traditional hospital follow-up played a 


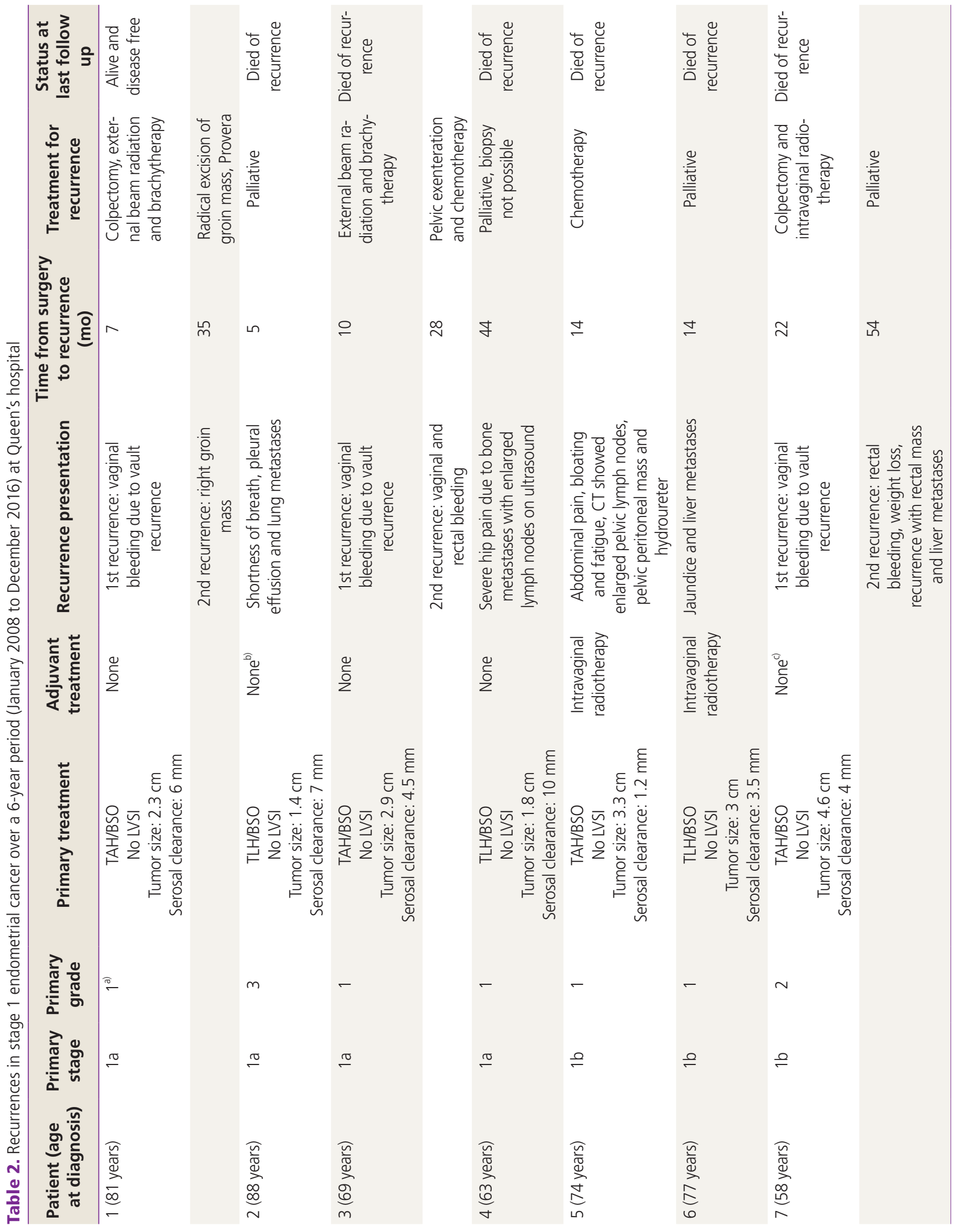




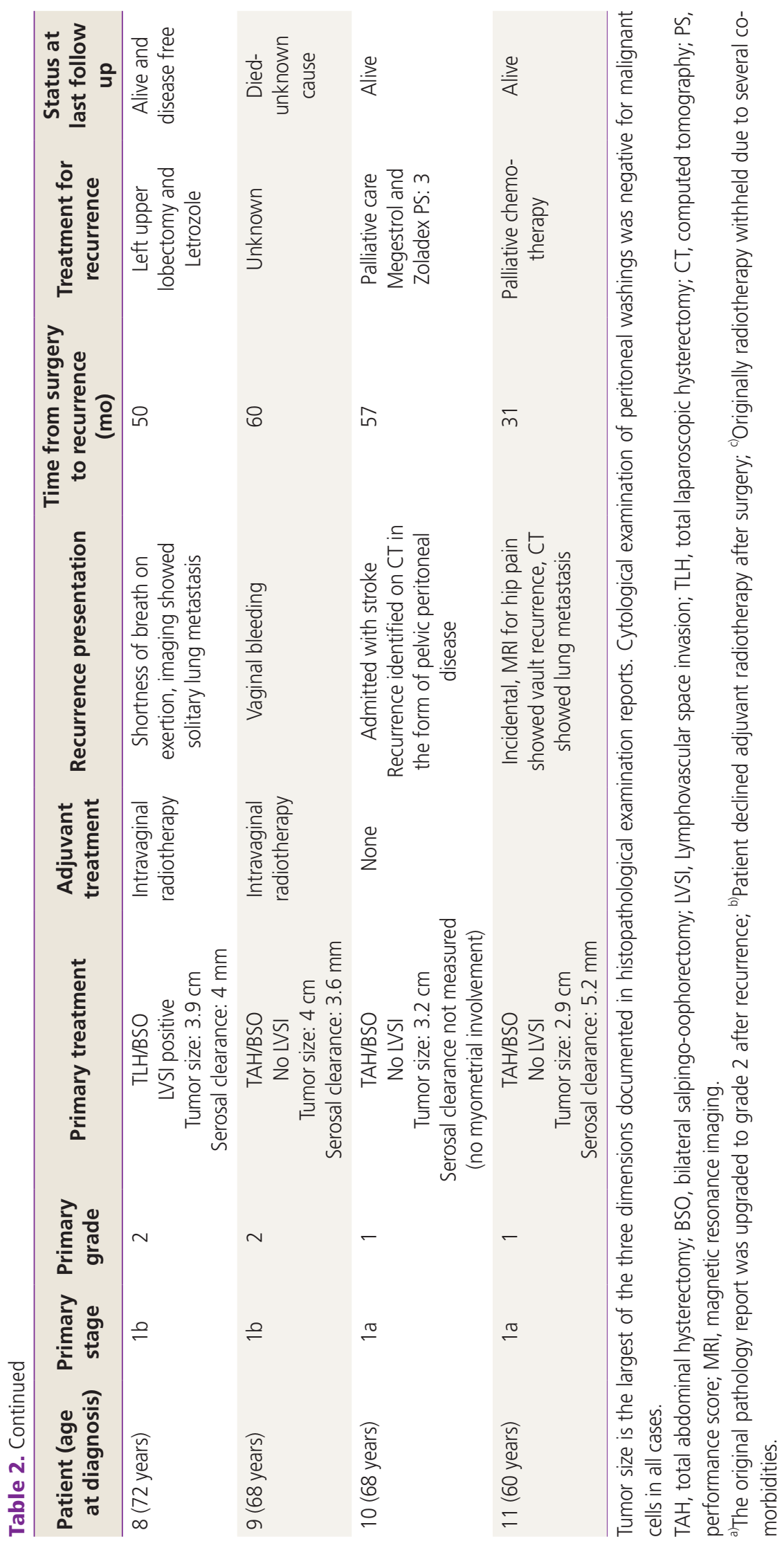




\section{Obstetrics \& Gynecology Science}

Manolis Nikolopoulos, et al. Endometrial cancer follow-up

limited role in the detection of disease recurrence. In 2 cases, the routine follow-up approach had a potential adverse effect on the patients, as both of them.

Waited for their routine clinic appointment scheduled a few weeks after the onset of symptoms. This illustrates the importance of the patients' education and understanding of the signs and symptoms of disease recurrence and the need for open communication pathways for the patient to initiate, if symptoms arise. In total, five patients had vaginal vault recurrence, four of them had bleeding, and one was asymptomatic. Two other patients experienced locoregional recurrence. None of these cases of recurrence were detected during the routine follow-up appointments and were either missed on clinical examination or became evident during the interval between the appointments. Furthermore, four patients with recurrence presented with distant metastatic disease, which was not detected during the routine follow-up appointments, questioning the effectiveness of routine pelvic examination for patients without vaginal bleeding.

In our study, none of the patients with early-stage endometrial cancers had asymptomatic recurrence identified during the routine follow-up appointments. A recent Danish study by Jeppesen et al. [14] found that $2.3 \%(61 / 2,612)$ of stage 1-2 endometrial cancers had an asymptomatic recurrence. Thus, 344 pelvic examinations need to be performed for asymptomatic recurrence. This is higher than the value that was previously reported in an older Norwegian study where one asymptomatic recurrence was detected for every 653 routine consultations for women with any stage disease who received curative surgical treatment, with no asymptomatic recurrences reported in women with stage $1 \mathrm{a}$ and $1 \mathrm{~b}$ disease, who were below the age of 60 years at the time of primary treatment [4]. There are limited comparable data regarding women in England. There is no evidence of a difference in outcome between the detection and subsequent treatment of a recurrence in asymptomatic and symptomatic patients [15-19], challenging an intensive follow-up strategy.

Regarding the pattern of recurrence in our study, four patients had distant recurrence $(1.3 \%)$ and 7 (2.3\%) had recurrence in the pelvis, vaginal vault, or pelvic peritoneum (locoregional). Recent studies have reported variable patterns depending on the risk factors, mainly on the histological subtype and initial staging [20-23]. For low risk for recurrence cancers (stage 1a, grade 1-2), locoregional recurrence rates are 2.1$6.2 \%$, while for intermediate-risk patients (stage 1a grade 3 , and stage $1 \mathrm{~b}$ with grade $1-2$ ) the rates are $2.3-9.2 \%$ [20-23].

The largest study till date reviewed all the patients with endometrial cancer in Germany between 2000 and 2016 [22]. Patients with a low risk for recurrence had a local recurrence rate of $3.5 \%$ and distant recurrence rate of $<1 \%$. Intermediate-risk patients had a local recurrence rate of $5.1 \%$ and distant recurrence rate of $2.8 \%$ for lung metastases, $2.3 \%$ for extrapelvic peritoneal metastasis, 1.8\% for liver, 1.8\% for brain, $1.5 \%$ for distal lymph nodes, and $1.3 \%$ for bone metastases. Low-risk patients had an incidence of recurrence with a peak between the fourth and sixth years, while the patients with intermediate risk had a peak incidence of recurrence within the first 3 years.

The studies by Jeppesen et al. [23] and Bendifallah et al. [20] reported a median time to recurrence of 12 and 13 months, respectively, for all the patients with stage 1 and stage 2 endometrial cancers. In our study, the median time to recurrence of stage 1 endometrioid endometrial cancer was 22 months.

The NHS is facing increasing financial pressures, with a need to develop more cost-effective ways of caring for patients to achieve sustainability in the future [8]. A recent randomized control trial (ENDCAT trial) assessed the patient acceptability and safety of nurse-led telephone versus hospital follow-up for women with stage 1 endometrial cancer and reported no detrimental physical or psychological outcomes for women with telephone follow-up compared to the traditional hospital follow-up $[24,25]$. Cost analysis of telephone follow-up compared to hospital follow-up was projected as cost-neutral for the NHS. However, a reduction in the clinical follow-up may provide more free appointments for other patients [26].

There is a shift to an alternative, more cost-effective followup for women with low risk for recurrence, early stage, endometrial cancer; examples being a nurse-led telephone follow-up [24] or a patient-initiated follow-up [27]. Patients with cancer should be treated according to their individual needs and the specific disease characteristics, as recommended by the UK's achieving world-class cancer outcomes report [28]. Stratified pathways for follow-up have already been implemented in breast, colorectal, and prostate cancer, including patient-initiated follow-up pathways [29], with patients finding self-management more convenient and timesaving than hospital follow-up and, more importantly, less stressful [30,31].

Patient-initiated follow-up (PIFU) aims to individualize pa- 


\title{
Obstetrics \& Gynecology Science
}

\author{
Vol. 64, No. 6, 2021
}

tient care based on the risk of recurrence and holistic needs, while simultaneously optimizing resources. A recent UK study reported that PIFU is well received by women treated for endometrial cancer, although younger women (median age 57 years) initiated more contact with the health care staff, indicating that they needed a higher level of support [32].

The NCSI 2013 advises that individuals are assessed to determine the tier of follow-up that would best meet their needs. Individuals deemed at low risk of recurrence and late effects (physical and psychosocial) are encouraged to opt for self-management, those at medium risk receive planned, coordinated care, and those at high risk receive complex care from specialist services. MDT review is required to triage the patients according to low, intermediate, or high risk of cancer recurrence [10]. Specific recommendations and eligibility criteria have recently been published by the British Gynaecological Cancer Society [33], and PIFU after the treatment of gynecological malignancy is already a routine part of the follow-up protocols in $42 \%$ (19/44) of the cancer centers in the UK according to a national study published in May 2020 [34].

A recent randomized control trial comparing traditional hospital follow-up and patient-initiated follow-up found that women in the patient-initiated group had significantly higher levels of fear of cancer recurrence than women receiving traditional hospital follow-up [27]. Although our study finds no value in the routine hospital appointments for detecting recurrence, regular contact with a healthcare provider may have psychological benefits for the patients. Qualitative data from the ENDCAT trial suggests that telephone clinic followup provides adequate support and information for women after the treatment of endometrial cancer; although the sample size of women interviewed was small, the results are favorable [25]. Further research in this area is required in the form of prospective studies to ensure that the psychological aspects of surviving cancer are addressed in the alternative modes of follow-up. Patient education regarding the various signs and symptoms of recurrence is of fundamental importance. In addition, the impact of background educational status and culture needs careful consideration. Rapid access to clinic appointments should be available for women with symptoms to provide appropriate timely care and support.

This has become even more relevant in the current time of the COVID-19 pandemic, as more patients have been followedup with telephone consultations in all hospitals across the UK.

\section{Weaknesses and future studies}

This study was limited by data availability due to the retrospective nature of this study. The small number of recurrence cases does not allow for potential correlations with demographics and risk factors to be revealed.

The number of patients with stage 1 grade 3 cancers was small, making this category unsuitable for deriving definite conclusions. Overall, this study mainly aimed at examining low-grade, stage 1 cancer recurrence, and the value of routine traditional follow-ups.

\section{Conclusion}

Our study has shown a low recurrence rate for stage 1, grade 1 , and grade 2 endometrioid endometrial adenocarcinoma, in line with the existing literature. Asymptomatic recurrences were not identified during routine follow-up appointments, despite a considerable amount of resources invested in the hospital follow-up appointments and clinical examinations. Our study supports the implementation of a stratified followup strategy with the understanding that larger studies are required in view of the low recurrence rate in early endometrial cancer.

\section{Conflict of interest}

No potential conflict of interest relevant to this article was reported.

\section{Ethical approval}

This study does not require approval of the Institutional Review Board because no patient identifiable data is contained in this article. The study was performed in accordance with the principles of the Declaration of Helsinki.

\section{Patient consent}

Written informed consent and the use of images from patients are not required for the publication. 


\section{Obstetrics \& Gynecology Science}

Manolis Nikolopoulos, et al. Endometrial cancer follow-up

\section{Funding information}

This research did not receive any specific grant from funding agencies in the public, commercial, or not-for-profit sectors.

\section{Acknowledgments}

The authors would like to thank Paula Lennon, Catherine Marling, Louise Dann, Katie Ayris, and Lois Delcher for collecting the data and note retrieval.

\section{References}

1. Duncan ME, Seagroatt V, Goldacre MJ. Cancer of the body of the uterus: trends in mortality and incidence in England, 1985-2008. BJOG 2012;119:333-9.

2. Ferlay J, Soerjomataram I, Dikshit R, Eser S, Mathers C, Rebelo $\mathrm{M}$, et al. Cancer incidence and mortality worldwide: sources, methods and major patterns in GLOBOCAN 2012. Int J Cancer 2015;136:E359-86.

3. Nevadunsky NS, Van Arsdale A, Strickler HD, Moadel A, Kaur G, Levitt J, et al. Obesity and age at diagnosis of endometrial cancer. Obstet Gynecol 2014;124:300-6.

4. Salvesen $H B$, Akslen LA, Iversen $T$, Iversen OE. Recurrence of endometrial carcinoma and the value of routine follow up. Br J Obstet Gynaecol 1997;104:1302-7.

5. Leeson S, Stuart N, Sylvestre Y, Hall L, Whitaker R. Gynaecological cancer follow-up: national survey of current practice in the UK. BMJ Open 2013;3:e002859.

6. Baekelandt MM, Castiglione M; ESMO Guidelines Working Group. Endometrial carcinoma: ESMO clinical recommendations for diagnosis, treatment and follow-up. Ann Oncol 2009;20 Suppl 4:29-31.

7. Tjalma WA, van Dam PA, Makar AP, Cruickshank DJ. The clinical value and the cost-effectiveness of followup in endometrial cancer patients. Int J Gynecol Cancer 2004;14:931-7.

8. Maddams J, Utley M, Møller H. Projections of cancer prevalence in the United Kingdom, 2010-2040. Br J Cancer 2012;107:1195-202.

9. Richards M, Corner J, Maher J. The National Cancer Survivorship Initiative: new and emerging evidence on the ongoing needs of cancer survivors. Br J Cancer
2011;105 Suppl 1:S1-4.

10. MacmillianCancer Support \& NHS Improvement. Living with and beyond cancer: taking action to improve outcomes. London: National Cancer Survivorship Initiative; 2013.

11. Mariani A, Webb MJ, Keeney GL, Haddock MG, Calori G, Podratz KC. Low-risk corpus cancer: is lymphadenectomy or radiotherapy necessary? Am J Obstet Gynecol 2000;182:1506-19.

12. Scholten AN, van Putten WL, Beerman H, Smit VT, Koper $\mathrm{PC}$, Lybeert ML, et al. Postoperative radiotherapy for stage 1 endometrial carcinoma: long-term outcome of the randomized PORTEC trial with central pathology review. Int J Radiat Oncol Biol Phys 2005;63:834-8.

13. Topfedaisi Ozkan N, Meydanlı MM, Sarı ME, Demirkiran F, Kahramanoglu I, Bese T, et al. Factors associated with survival after relapse in patients with low-risk endometrial cancer treated with surgery alone. J Gynecol Oncol 2017;28:e65.

14. Jeppesen MM, Mogensen $O$, Hansen DG, lachina $M$, Korsholm M, Jensen PT. Detection of recurrence in early stage endometrial cancer-the role of symptoms and routine follow-up. Acta Oncol 2017;56:262-9.

15. Shumsky AG, Stuart GC, Brasher PM, Nation JG, Robertson DI, Sangkarat $\mathrm{S}$. An evaluation of routine follow-up of patients treated for endometrial carcinoma. Gynecol Oncol 1994;55:229-33.

16. Agboola OO, Grunfeld E, Coyle D, Perry GA. Costs and benefits of routine follow-up after curative treatment for endometrial cancer. CMAJ 1997;157:879-86.

17. Gadducci A, Cosio S, Fanucchi A, Cristofani R, Genazzani AR. An intensive follow-up does not change survival of patients with clinical stage I endometrial cancer. Anticancer Res 2000;20:1977-84.

18. Fung-Kee-Fung M, Dodge J, Elit L, Lukka H, Chambers A, Oliver $T$, et al. Follow-up after primary therapy for endometrial cancer: a systematic review. Gynecol Oncol 2006;101:520-9.

19. Sartori E, Pasinetti B, Chiudinelli F, Gadducci A, Landoni $F$, Maggino $T$, et al. Surveillance procedures for patients treated for endometrial cancer: a review of the literature. Int J Gynecol Cancer 2010;20:985-92.

20. Bendifallah S, Ouldamer L, Lavoue V, Canlorbe G, Raimond $E$, Coutant $C$, et al. Patterns of recurrence and outcomes in surgically treated women with endometrial 


\section{Obstetrics \& Gynecology Science}

Vol. 64, No. 6, 2021

cancer according to ESMO-ESGO-ESTRO Consensus Conference risk groups: results from the FRANCOGYN study Group. Gynecol Oncol 2017;144:107-12.

21. Francis $S R$, Ager BJ, Do OA, Huang YJ, Soisson AP, Dodson $\mathrm{MK}$, et al. Recurrent early stage endometrial cancer: patterns of recurrence and results of salvage therapy. Gynecol Oncol 2019;154:38-44.

22. Ignatov $T$, Eggemann $H$, Costa SD, Ortmann O, Ignatov A. Endometrial cancer subtypes are associated with different patterns of recurrence. J Cancer Res Clin Oncol 2018;144:2011-7.

23. Jeppesen MM, Jensen PT, Gilså Hansen D, lachina M, Mogensen $\mathrm{O}$. The nature of early-stage endometrial cancer recurrence-A national cohort study. Eur J Cancer 2016;69:51-60.

24. Beaver K, Williamson S, Sutton C, Hollingworth W, Gardner A, Allton B, et al. Comparing hospital and telephone follow-up for patients treated for stage-I endometrial cancer (ENDCAT trial): a randomised, multicentre, non-inferiority trial. BJOG 2017;124:150-60.

25. Williamson S, Beaver K, Gardner A, Martin-Hirsch P. Telephone follow-up after treatment for endometrial cancer: a qualitative study of patients' and clinical nurse specialists' experiences in the ENDCAT trial. Eur J Oncol Nurs 2018;34:61-7.

26. Dixon P, Beaver K, Williamson S, Sutton C, Martin-Hirsch $P$, Hollingworth W. Cost-consequence analysis alongside a randomised controlled trial of hospital versus telephone follow-up after treatment for endometrial cancer. Appl Health Econ Health Policy 2018;16:415-27.

27. Jeppesen MM, Jensen PT, Hansen DG, Christensen RD, Mogensen $\mathrm{O}$. Patient-initiated follow up affects fear of recurrence and healthcare use: a randomised trial in early-stage endometrial cancer. BJOG 2018;125:1705-14. 28. Independent Cancer Task Force. Achieving worldclass cancer outcomes: a strategy for England 2015-2020. [Internet]. London (UK): National Health Service; c2020 [cited 2021 Jan 15]. Available from: https://www.england.nhs.uk/wp-content/uploads/2016/10/cancer-oneyear-on.pdf.

29. Watson EK, Rose PW, Neal RD, Hulbert-Williams N, Donnelly $P$, Hubbard $G$, et al. Personalised cancer follow-up: risk stratification, needs assessment or both? $\mathrm{Br} J$ Cancer 2012;106:1-5.

30. Kimman ML, Bloebaum MM, Dirksen CD, Houben RM, Lambin P, Boersma LJ. Patient satisfaction with nurse-led telephone follow-up after curative treatment for breast cancer. BMC Cancer 2010;10:174.

31. Williamson S, Chalmers K, Beaver K. Patient experiences of nurse-led telephone follow-up following treatment for colorectal cancer. Eur J Oncol Nurs 2015;19:237-43.

32. Kumarakulasingam $P$, McDermott $H$, Patel $N$, Boutler $L$, Tincello DG, Peel $D$, et al. Acceptability and utilisation of patient-initiated follow-up for endometrial cancer amongst women from diverse ethnic and social backgrounds: a mixed methods study. Eur J Cancer Care (Engl) 2019;28:e12997.

33. Newton C, Nordin A, Rolland $P$, Ind $T$, Larsen-Disney $P$, Martin-Hirsch $P$, et al. British Gynaecological Cancer Society recommendations and guidance on patient-initiated follow-up (PIFU). Int J Gynecol Cancer 2020;30:695-700.

34. Coleman L, Newton C. Patient initiated follow up after gynaecological malignancy: national survey of current UK practice. Eur J Obstet Gynecol Reprod Biol 2020;248:193-7. 